\title{
Cluster Detection and Quantification
}

\author{
D.J. Larson* and M.K. Miller** \\ * Imago Scientific Instruments, Madison, WI 53711 \\ ** Materials Science and Technology Division, Oak Ridge National Laboratory, PO Box 2008, Oak \\ Ridge, TN 37831-6136
}

The properties of many materials are defined by the presence of small precipitates or clusters of individual atoms. In principle, atom probe tomography (APT) has sufficient spatial resolution to detect the smallest precipitates so that the early stages of phase separation can be experimentally investigated. "Friends-of-friends"-type analysis methods [1-3] have been developed to detect clusters and quantify the size, number density and composition of these precipitates in dilute solid solutions. In this study, the influence of cluster radius, cluster composition, and instrument detection efficiency [4] on the detection and quantification of ultra-fine clusters/precipitates was examined.

In order to perform a systematic study, the desired microstructures were constructed with an atom probe simulator. It is advantageous to use simulated data to obtain a metric (in this case, percentage error) for cluster detection, especially for very small clusters. The parameters used for the simulations were an $\alpha$-Fe body centered cubic matrix with $\sim 0.02 \%$ solute containing $6.3 \times 10^{23} \mathrm{~m}^{-3}$ of coherent $0.25,0.5$ and $1.0 \mathrm{~nm}$ radius clusters $(\sim 5, \sim 42$, and $\sim 350$ atoms/cluster, respectively) containing either 50 or $100 \%$ solute $(\mathrm{Cu})$ atoms. As typical APT detector efficiencies range from $\sim 35 \%$ to $\sim 65 \%$, the detection efficiency was varied between 30 and $100 \%$ by randomly removing the appropriate fraction of the simulated atoms. A 3-dimensional $0.1 \mathrm{~nm}$ Gaussian distributed scatter was applied to the atom positions to simulate trajectory aberrations [4]. The simulated data, shown in Fig. 1 (atom maps of $0.25,0.5$ and $1 \mathrm{~nm}$ radius precipitates containing $50 \%$ or $100 \%$ solute with $50 \%$ detector efficiency), are 40 by 40 by $40 \mathrm{~nm}$ volumes containing 65 clusters. These data were then analyzed with the maximum separation envelope method with a maximum separation distance of $d_{\max }=0.5 \mathrm{~nm}$ [1] to attempt to detect the clusters. Because some of the data contain clusters with very few atoms $(<5)$, cluster detection was considered to occur if a cluster of two or more atoms was detected. Although a variety of cluster detection algorithms exist [1-3], only one method was used, which focused on a yes/no detection of clusters as opposed to an estimation of the quantitative composition based on the numbers of atoms detected per cluster.

The error percentage (defined as the absolute value of the difference between the known and the detected cluster counts, normalized by the known count) as a function of detector efficiency is shown in Fig. 2. For all efficiencies simulated, detection of the clusters is obtained for all clusters $\geq 0.5 \mathrm{~nm}$ radius. For the smallest clusters, the correct number of clusters is never detected, regardless of efficiency. A contour plot of error, Fig. 3, highlighting the low cluster radius/efficiency regime provides a useful estimate of expected error [5].

[1] J. M. Hyde and C. A. English, in: Proc. MRS 2000 Fall Meeting, Sym. R, Boston, MA, Nov.27-30, 2000, MRS, Pittsburgh, PA, vol. 650 (2001) R6.6.1.

[2] D. Vaumousse, P. J. Warren and A. Cerezo, Mat. Sci. Engr. A 353 (2003) 215.

[3] L. T. Stephenson et al., Micro. Microanal. 13 (2007) 448.

[4] M. K. Miller, A. Cerezo, M. G. Hetherington and G. D. W. Smith, Atom Probe Field Ion Microscopy, (1996) Oxford University Press.

[5] Research at the Oak Ridge National Laboratory SHaRE User Facility was sponsored by the Scientific User Facilities Division, Office of Basic Energy Sciences, U.S. Department of Energy. 

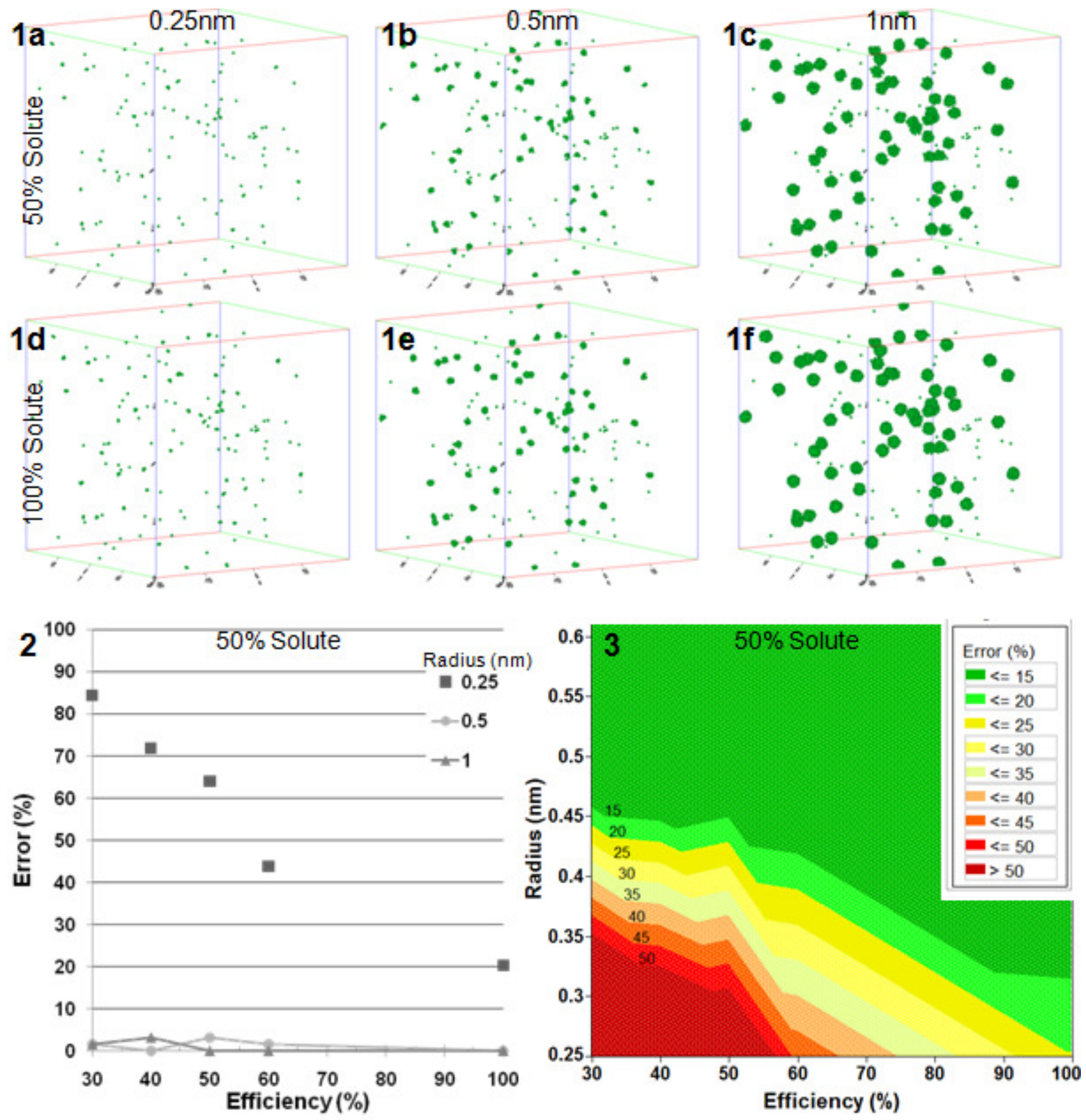

FIG. 1. Simulated data sets of solute clusters generated with $50 \%$ instrument efficiency and a) $0.25 \mathrm{~nm}$ radius with $50 \%$ solute, b) $0.5 \mathrm{~nm}$ radius with $50 \%$ solute, c) $1.0 \mathrm{~nm}$ radius with $50 \%$ solute, d) $0.25 \mathrm{~nm}$ radius with $100 \%$ solute, e) $0.5 \mathrm{~nm}$ radius with $100 \%$ solute and f) $1.0 \mathrm{~nm}$ radius with $100 \%$ solute. Each volume is $40 \mathrm{~nm}$ by $40 \mathrm{~nm}$ by $40 \mathrm{~nm}$ and contains 65 clusters.

FIG. 2. Detection error for $0.25,0.5$ and $1.0 \mathrm{~nm}$ precipitates as a function of instrument efficiency. FIG. 3. Contour plot of detection error as a function of instrument efficiency and precipitate radius. 